\title{
Effective Use of Organisational Abstractions for Confidence Models ${ }^{1}$
}

\author{
Ramón Hermoso Holger Billhardt Roberto Centeno Sascha Ossowski \\ Artificial Intelligence Group, DATCCCIA, University Rey Juan Carlos \\ C/Tulipán s/n, 28937, Móstoles (Madrid), Spain \\ \{ramon.hermoso, holger.billhardt, roberto.centeno, sascha.ossowski\}@urjc.es
}

\section{Introduction}

Trust and reputation systems are not only useful for Virtual Organisations (VOs), but VOs also for trust and reputation mechanisms. The structure provided by a VO can be used to construct more effective trust mechanisms. In particular, the structural elements defined in a VO (e.g., roles and interactions) provide a certain notion of similarity which allows agents to infer the expected behaviour of acquaintances within new situations by analysing their past behaviour within similar situations. This property is especially useful in situations where agents do not yet have sufficient experiences related to a specific type of interaction, or within very volatile environments.

In this paper [1] we continue our previous work [2] on trust mechanisms for VOs. We present some experiments that show how the use of organisational abstractions can effectively improve trust mechanisms. We also put forward a testbed developed to compare trust models in VOs (TOAST).

\section{Confidence and Trust for Organisational Structures}

As outlined in [2], it is natural to consider that agents participating in a VO play some roles in different interactions. In addition, we assume that the agents know the organisational structure, e.g., they know the existing roles and interaction types, the roles that participate in each interaction type, as well as the roles other agents are playing within the organisation.

Similarly to other approaches [3, 4], we build our trust model on the idea of confidence and reputation. Both are ratings agents use in order to evaluate the trustworthiness of other agents in a particular issue (e.g., playing a particular role $\mathrm{R}$ in a particular interaction $\mathrm{I}$ ). Confidence $c_{A \rightarrow\langle B, R, I\rangle}$, confidence that agent $A$ has on agent $B$ playing role $R$ in interaction $I$ - is a local measure that is only based on an agent's own past experiences, while reputation is an aggregated value an agent gathers by asking its acquaintances about their opinion regarding the trustworthiness of another agent. Thus, reputation can be considered as an external or social measure. We define trust $-t_{A \rightarrow\langle B, R, I\rangle}$ - as a rating resulting from combining confidence and reputation values.

Agents will accumulate their previous experiences in their internal structures we call local interactions table (LIT). When only a few experiences have been performed previously, classical approachs suggest to use reputation mechanisms in order to gather opinions about trust others have about a third party. However, this view poses some problems, namely opinions from $m a-$ licious agents. Thus, our approach proposes agents to take into account similiar past situations in order to infer behaviours of others [2]. This approach relies on the hypothesis that, in general, agents behave in a similar way in all interactions related to the same role. We argue that, exploiting this idea, the more similar $I^{\prime}$ and $I$ are, the more similar the values $c_{A \rightarrow\left\langle B, R, I^{\prime}\right\rangle}$ and $c_{A \rightarrow\langle B, R, I\rangle}$ will be. The same applies to roles. Using this assumption, confidence ratings accumulated for similar agent/role/interaction tuples may provide evidence for the trustworthiness of the issue $\langle B, R, I\rangle$. Based on this idea, we propose to build trust by taking into account all the past experiences an agent has, focusing on their degree of similarity with the issue $\langle B, R, I\rangle$. In particular, we calculate

\footnotetext{
${ }^{1}$ The present work has been funded by the Spanish Ministry of Education and Science under project TIC200308763-C02-02.
} 
trust as a weighted mean over all the confidence values an agent has accumulated in its LIT. This is shown in the following equation:

$$
t_{A \rightarrow\langle B, R, I\rangle}=\frac{\sum_{\langle X, Y, Z\rangle \in L I T_{A}} c_{A \rightarrow\langle X, Y, Z\rangle} \cdot r_{A \rightarrow\langle X, Y, Z\rangle} \cdot \operatorname{sim}(\langle X, Y, Z\rangle,\langle B, R, I\rangle)}{\sum_{\langle X, Y, Z\rangle \in L I T_{A}} r_{A \rightarrow\langle X, Y, Z\rangle} \cdot \operatorname{sim}(\langle X, Y, Z\rangle,\langle B, R, I\rangle)}
$$

where the entries in agent A's LIT are used in order to estimate the confidence agent A has on issue $\langle X, Y, Z\rangle$ by combining confidence reliability $\left(r_{A \rightarrow\langle X, Y, Z\rangle}\right)$ and the similarity of the issue $\langle X, Y, Z\rangle$ to the target issue $\langle B, R, I\rangle$. We suppose that organisational models include information from which issues similarities can be derived.

\section{Trust Organisational Agent System Testbed (TOAST)}

TOAST is a tool we have developed in order to test our proposed model and to provide evidence that our assumptions are correct. TOAST evaluates the influence of different trust models on the evolution of the overall utility of an agent or a society of agents. This testbed simulates a virtual organisation where agents have to interact with others in order to achieve their goals. In our experiments we used a virtual organisation with 20 agents (with same behaviour) and the testbed generated randomly 40000 goals for those 20 agents. We have tested three different models: a random model, a past history-based model and our inference model based on similarities. The results confirm that the use of organisational structures makes agents' decision-making easier and more efficient, in particular when agents have very few previous experiences within the organisation.

\section{Conclusion}

In this paper we have presented results of our work, aimed at integrating trust mechanisms into virtual organisations. We have tackled the problem of locally calculating trust, that is, finding "good" counterparts, even if only very few previous experiences are available and without the need of using reputation information obtained from external sources. The proposed model takes into account key concepts of organisational models, such as roles and interactions. It has confidence inference capabilities exploiting organisational structures provided by VOs. We have tested our model, confirming that the use of organisational structures makes agents' decision-making easier and more efficient, in particular when agents join an organisation and, thus, can not count on their own previous experiences. Furthermore, we have presented TOAST, the testbed that we have developed to test our assumptions.

\section{References}

[1] Ramón Hermoso, Holger Billhardt, Roberto Centeno, and Sascha Ossowski. Effective use of organisational abstractions for confidence models. In Michael O'Grady Oguz Dikenelli Gregory O'Hare, Alessandro Ricci, editor, 7th Annual International Workshop Engineering Societies in the Agents World, pages 246-261, September 2006.

[2] Ramón Hermoso, Holger Billhardt, and Sascha Ossowski. Integrating trust in virtual organisations. In AAMAS-06 Workshop on Coordination, Organization, Institutions and Norms in agent systems (COIN), pages 121-133, 2006.

[3] T. Dong Huynh, Nicholas R. Jennings, and Nigel R. Shadbolt. FIRE: An integrated trust and reputation model for open multi-agent systems. In Proceedings of the 16th European Conference on Artificial Intelligence (ECAI), 2004.

[4] Jordi Sabater and Carles Sierra. Reputation and social network analysis in multi-agent systems. In AAMAS '02: Proceedings of the first international joint conference on Autonomous agents and multiagent systems, pages 475-482, New York, NY, USA, 2002. ACM Press. 